\title{
AUTOMATIC RAILWAY POWER LINE EXTRACTION USING MOBILE LASER SCANNING DATA
}

\author{
Shanxin Zhang ${ }^{\mathrm{a}, \mathrm{b}}$, Cheng Wang ${ }^{\mathrm{a}, *}$, Zhuang Yang ${ }^{\mathrm{a}}$, Yiping Chen ${ }^{\mathrm{a}}$, Jonathan $\mathrm{Li}^{\mathrm{a}, \mathrm{c}}$ \\ ${ }^{a}$ Fujian Key Laboratory of Sensing and Computing for Smart Cities, School of Information Science and Engineering,Xiamen, FJ 361005, \\ China - handsomeshanxin@163.com,cwang@xmu.edu.cn,hongyiliao@163.com,ypchenhk@gmail.com \\ ${ }^{\mathrm{b}}$ Xizang Key Laboratory of Optical Information Processing and Visualization Technology, Information Engineering College, \\ Xizang Minzu University, Wenhuidong Street, Xianyang,SX 712082, China - handsomeshanxin@163.com \\ ${ }^{c}$ Mobile Mapping Lab, Department of Geography and Environmental Management, University of Waterloo, \\ Waterloo, ON N2L 3G1, Canada - junli@uwaterloo.ca
}

Commission V, WG V/3

KEY WORDS: Power line extraction, Mobile laser scanning, Quaternion algorithm, Hough voting, Point cloud

\begin{abstract}
:
Research on power line extraction technology using mobile laser point clouds has important practical significance on railway power lines patrol work. In this paper, we presents a new method for automatic extracting railway power line from MLS (Mobile Laser Scanning) data. Firstly, according to the spatial structure characteristics of power-line and trajectory, the significant data is segmented piecewise. Then, use the self-adaptive space region growing method to extract power lines parallel with rails. Finally use PCA (Principal Components Analysis) combine with information entropy theory method to judge a section of the power line whether is junction or not and which type of junction it belongs to. The least squares fitting algorithm is introduced to model the power line. An evaluation of the proposed method over a complicated railway point clouds acquired by a RIEGL VMX450 MLS system shows that the proposed method is promising.
\end{abstract}

\section{INTRODUCTION}

Railway power supply security is closely related to our everyday life and industrial activities. Power interruptions may lead to seriously railway traffic accident. The inspection of railway power line is an important work of railway maintenance management departments. In order to prevent and eliminate railway traffic accident caused by power interruptions and ensure the safety of the operation of electric power line, railway maintenance management department need to spend a lot of manpower and material resources to inspect power line every year. Currently, with the rapid development of remote sensing technology, remote sensing technology has been used more and more widely in power line extraction. Most of utility companies are endeavouring to find the more cost-effective techniques to obtain clearances and improve access to the power lines, which could be achieved by using the latest state-of-the-art remote sensing equipment such as digital camera and LiDAR system.

In recent years, many research works have been carrying out using Airborne LiDAR data for automatic detection and extraction of power lines. They applied many valid methods to reconstruct 3D power lines using airborne LiDAR point clouds such as the Random Sample Consensus (RANSAC) method (Chen et al., 2012), Markov Random Field (MRF) classifier (Sohn et al., 2012), Hough Transform method (Liu et al., 2009) and Random Forests classification method (Kim and Sohn, 2013). But there two defects exist in airborne laser scanning system lead to it impossible implement very well in railway power line detection. On the one hand, although the recent airborne laser scanning system is being applied in power line detection, few companies can afford such a cost. On the other hand, its no way to access to the point cloud data to detect the power line under the circumstance

\footnotetext{
${ }^{*}$ Corresponding author
}

of in railway station and in tunnel.

MLS system is developed rapidly in recent years. It has a unique advantage in the quick access to the ground surface information. And furthermore, MLS can produce extremely higher density point clouds, generating digital elevation models with $\mathrm{cm}$-level resolution, while most of ALS systems only have a $50 \mathrm{~cm}$-level resolution. Research on mobile laser point cloud data in power line extraction technology has important practical significance on railway power lines patrol work. (Lehtomäki et al., 2010) proposed an automatic method to extract pole-like objects from ML$\mathrm{S}$ data. (Cheng et al., 2014) presented a method to extract power line points using a voxel-based hierarchical method, and then the point densities of neighboring voxels are calculated for further filtering. (Guan et al., 2014) proposed to extract power-line points in the identified non-road points, followed by Hough transform and Euclidean distance clustering.

There are a lot of lines in railway traffic circumstance, only part of them is power lines. In previous research, those methods above cannot fit complex railway surroundings very well. They cannot distinct the power line from the vertical suspension wire and horizontal suspension wire in the wire net and cannot find out the joint region of the power line either. In this paper, we propose a stepwise method to extract the power line feasible and fast enough. This method use spatial information between power line and trajectory to separate a segment non-road points from original point cloud data. Then, use the self-adaptive space region growing method to extract lines parallel with rails. Finally use PCA and information entropy theory to get the power line points. We also build three dimensional (3D) model of power line use the least squares fitting algorithm. The proposed algorithm has been tested on a MLS dataset acquired by a RIEGL VMX-450 system. 


\section{ACUISITION}

This section includes two parts. One part is presentation the mobile laser scanning system, the other part is to explain the data source.

\subsection{Mobile laser scanning system}

MLS system uses car as remote sensing platform and comprises four parts: (1) a high-precision dynamic Global Navigation Satellite System (GNSS), (2) high dynamic carrier posture sensor Inertial Measurement Unit (IMU), (3) fully-integrated calibrated laser scanners, (4) optional camera sub-system. GNSS/IMU component provides positioning informant to sensor and makes the vehicle-mounted system has the ability of direct location. Laser scanner can scan out with a high density of point cloud data. Optional camera provides color information to point cloud data.

The RIEGL VMX-450 Mobile Laser Scanning System (as Figure 1 shows) offers extremely high measurement rates providing dense, accurate, and feature-rich data even at high driving speeds. It can apply to fast 3D data collection, featuring high accuracy and high resolution, provides a basis for a variety of applications like mapping of roadways and rail corridors.

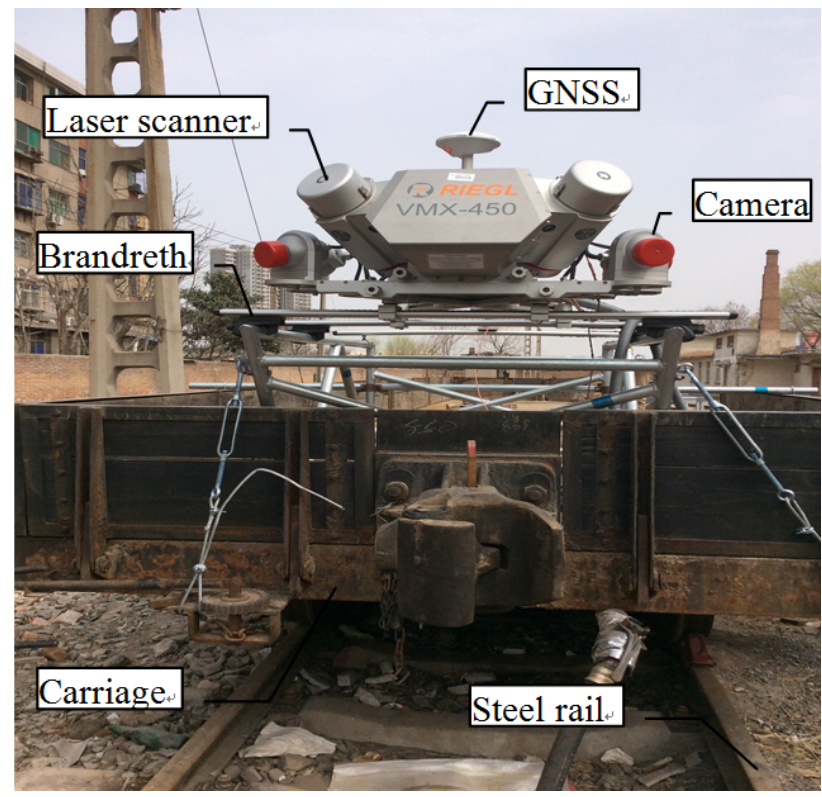

Figure 1. RIEGL VMX-450 system

\subsection{Data Resources}

The data resources were acquired by a RIEGL VMX-450 system which installed on the train. The train was traveling at 120 miles an hour when scanning the scene. The point density is approximately 3000 points per square meter. The point cloud data is shown as Figure 2. The power lines point density is very big. Through this picture we can see that the power lines in the real surrounding is very complex.

\section{METHOD}

There are some fixed priors that are fit to the extraction of power line points in railway circumstance. For example, power lines,

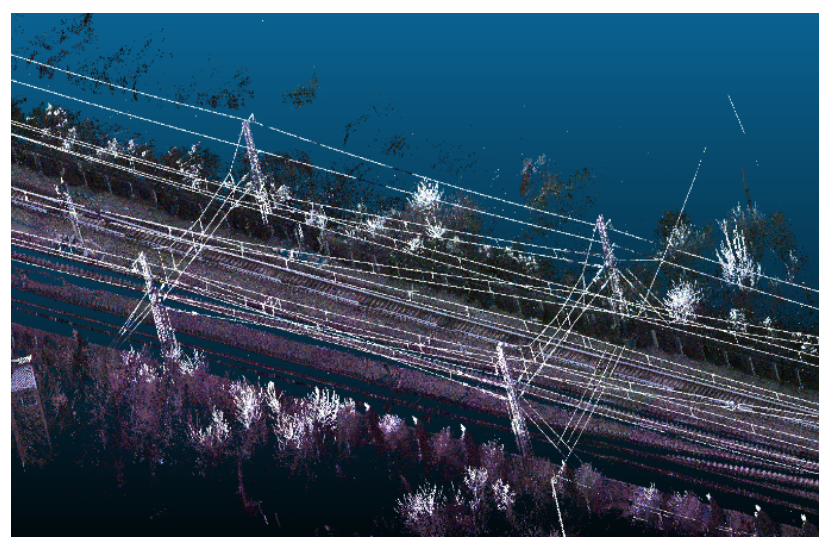

Figure 2. Railway power line point cloud data

horizontal suspension lines and trajectory parallel with rail; power line and horizontal suspension line appears in pairs and the horizontal suspension line above the power line; the power line above the trajectory and so on.

The proposed method follows three steps: significant point cloud data segment, power line extraction, joint region judgement.

\subsection{Point cloud segment}

The point cloud segment is very important. We can filter out the ground point by this step.

In order to obtain the width of power line area, we use feature of that there are protective fences between edge of railways and protective fences elevation higher than trajectory (as Figure 3 shows). The trajectory coordinates are known. We can get width of significant point cloud which is perpendicular to trajectory.

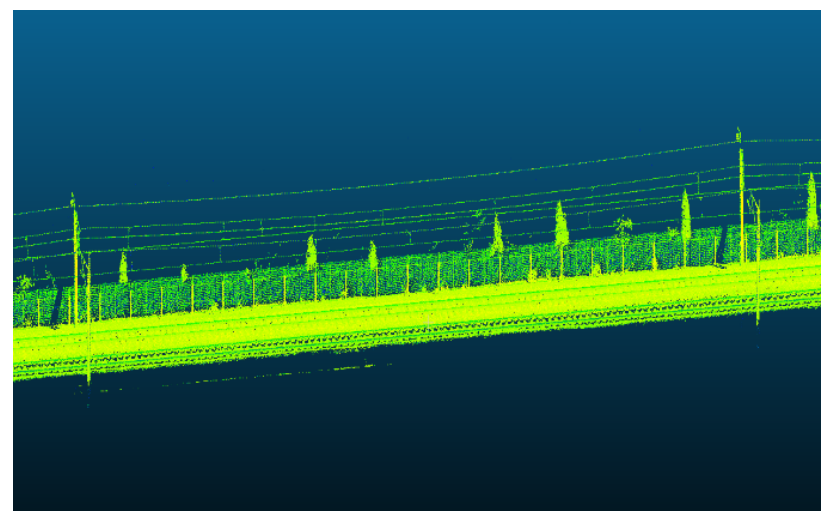

Figure 3. Protective fence

Note that, trajectory about $2 \mathrm{~m}$ above rails. Through the feature that horizontal suspension wire power line is located more than $4 \mathrm{~m}$ above the trajectory according to the power line construction standards (between $5.7 \mathrm{~m}$ to $6.5 \mathrm{~m}$ in china), we got the height range of the power line.

A bound box can be built by the trajectory length, width and height of power line area. Using the bounding box method read significant point in the point cloud data with octree data structure. 
The significant point cloud data extraction result as shown in Figure 4.

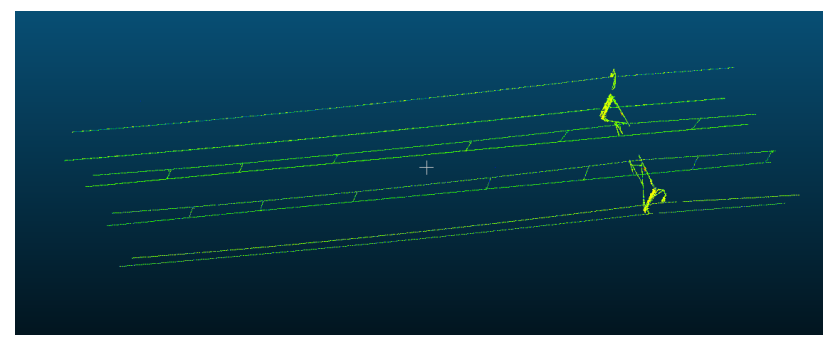

Figure 4. Result of significant point cloud segmentation

\subsection{Power line extraction}

In order to automatic and fast extraction the power line in complex rail circumstance, we presented an algorithm of self-adaptive space region growing method.

The algorithm idea of self-adaptive space region growing method as follows. Generate seeds. Select a point as seed and a growth direction in the initial position of cloud. Generate a cross section according seed and its direction and make it to grow at a scalable length. Using PCA and information entropy to determine the label of the power line point cloud which is included in the growth space. Generate a new seed and a new direction overlap it again until this seed end. Select the other seed until all seeds have been selected. The process flow of point line extraction as shown in Figure 5.

First of all, we should generate the seeds and their corresponding directions. The detailed steps are as follows.

1. Acquire a section point cloud $p_{s}$ along trajectory form segmentation point cloud $p_{\text {seg }}$ and rotate it to a vertical plane which normal vector is trajectory direction. We label the vertical plane with $p_{g}$ and mark the rotated point cloud with $p_{r}$. Divide grid in $p_{g}$ and project $p_{r}$ into $p_{g}$.

2. Select a grid when the number of points in a grid greater than a threshold. Add those points into array $P L[]$.

3. Select $n$ points which has maximum $\mathrm{n}$ values in $\mathrm{Z}$ axis (trajectory direction) in $P L[]$. Compute the central position of those points to generate a new point as seed $s_{i}$ and add $s_{i}$ into $S$ []. If two grid both include seed and one grid above another grid in vertical direction, then the seed in down grid is power line seed, label its line kind with power line. The seed in upon grid is horizontal suspension wire seed, label its line kind with horizontal suspension wire.

4. Select $\mathrm{n}$ points which has minimum $n$ values in $\mathrm{Z}$ axis (trajectory direction) in $P L[]$. Compute the central position of those points to generate a new point $r_{i}$. Add the direction of $r_{i}$ to $s_{i}$ into direction array $D[]$.

5. Jump into step 2 until all grids which points greater than a threshold are computed.

Then, we select one seed and its direction to extract a power line and find out the junction area witch joint with vertical suspension line in the power line.

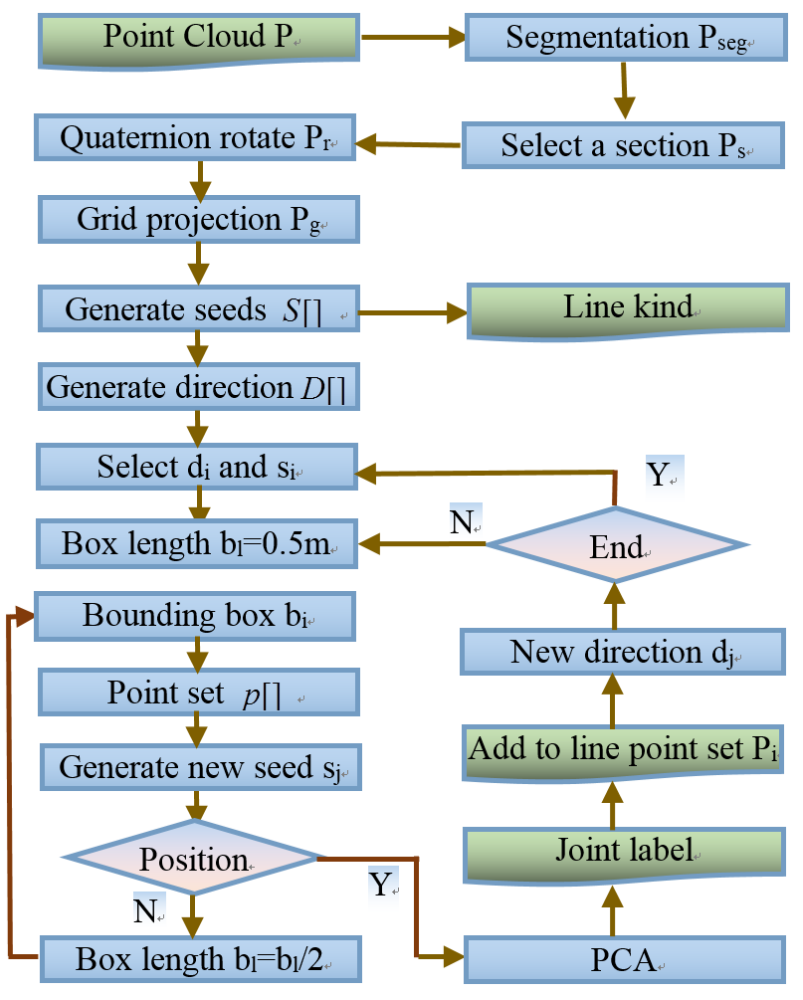

Figure 5. Point line extraction flow

1. Select a seed $s_{i}$ and its direction $d_{i}$.

2. Initialize the box length $b_{l}$ equals a value such as $0.4 \mathrm{~m}$.

3. Use seed point as central position to generator a square which side length equals $a$. The square represent a planar model, and together with a given length bl, generates a 3D polygonal bounding box $b_{i}$. The bounding box is then used to segment all points lying inside it. We store those points in point set $p[]$.

4. Obtain points in a quarter of box length in the last part. If number of points not equal to zero, then compute the centre of those points to generate new seed $s_{j}$ and jump into step 4 , else set box length $b_{l}=b_{l} / 2$, jump into step 3 .

5. Set box length $b_{i}=0.5$. Use PCA method to judge the point in $p[]$ whether is line or joint and give it corresponding labels. Add $p[]$ to line point set $p_{i}$.

6. Use the direction $s_{i}$ to $s_{j}$ generate a new direction $d_{j}$.

7. If the distance between $s_{i}$ and $s_{j}$ smaller than a threshold, then this power line extracted over, else jump into step 2 .

Select other seed and its corresponding direction to extract its corresponding line use step 1 to step 7 until $S[]$ equals null. Seed set $S[]$ equals null means that all power lines and horizontal suspension lines has been extracted. And in this progress, we labelled every joint position and its joint type. Section 3.3 explain how do we classify the joint labels.

\subsection{Joint region judgement}

The main function of this part is to label point $p_{k}$ in $p[]$ which segmented by bounding box $b_{i}$. We define three labels in our method as Table 1 shown. This part include two sides, one is use PCA method to compute eigenvectors of points segmented 
by bounding box

\begin{tabular}{|l|c|}
\hline Labels & Description \\
\hline$L[1]$ & $p_{k}$ straight power line \\
$L[2]$ & $p_{k}$ 2D suspension joint \\
$L[3]$ & $p_{k}$ 3D suspension joint \\
\hline
\end{tabular}

Table 1. Description of labels

3.3.1 Use PCA extracts space features: PCA is referred to as a dimensionality reduction algorithm. It tries to identify the subspace in which the data approximately lies. PCA will do more directly, and will require only an eigenvector calculation of covariance matrix (Guebel and Torres, 2013). In this application, covariance matrix has three eigenvalues in eigenvector.

Select one point $p_{i}$ from point set $p[]$. Use $p_{i}$ as centre and radius $a$ make a sphere $s p h_{i}$. The sphere centre is a candidate joint centre. The points include in the sphere are candidate joint points. Use $s p h_{i}$ to select points from $p$ []. Apply PCA algorithm on those selected point we can get three eigenvalues: $\lambda_{1}^{i}, \lambda_{2}^{i}, \lambda_{3}^{i}$.

1. If $\lambda_{1}^{i}$ is much larger than $\lambda_{2}^{i}, \lambda_{3}^{i}$, the points in $s p h_{i}$ show the 1D linear features. The point $p_{i}$ should be labelled as $L[1]$.

2. If $\lambda_{1}^{i}, \lambda_{2}^{i}$ is much larger than $\lambda_{3}^{i}$, the points in $s p h_{i}$ show the 2D surface features. The point $p_{i}$ should be labelled as $L[2]$.

3. If $\lambda_{1}^{i}, \lambda_{2}^{i}, \lambda_{3}^{i}$ almost have the same size, the points in $s p h_{i}$ show the $3 \mathrm{D}$ spatial features. The point $p_{i}$ should be labelled as $L[3]$.

The size of the eigenvalue is related to the location of $s p h_{i}$ and the size of the radius of $s p h_{i}$. As in Figure 6 (a) shows, $p_{1}$ and $p_{2}$ has different position, we have $\lambda_{1}^{1}<\lambda_{1}^{2}$, this result in accordance with $p_{1}$ has less linear feature than $p_{2}$ in the figure. As in Figure 6 (b) shows, $p_{2}$ as the sphere centre, the sphere has different radius, we have $\lambda_{1}^{1}<\lambda_{1}^{2}$, this result shows that $p_{2}$ represent more linear feature in a larger area in accordance with figure described.
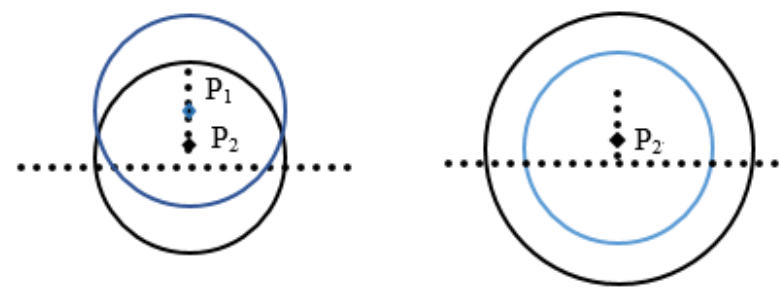

Figure 6. Eigenvalue changing in different location. (a) Sphere center is different. (b) Sphere radius is different.

We should select the most appropriate position as joint position. In order to select the accurate joint position, we use information entropy to evaluate the difference of those similar eigenvalue distribution. For a point, we judge which joint label class it belongs to according to its entropy value in point set $p[]$. How to compute entropy value and how to use entropy value to classify the joint kind will be illustrated in section 3.3.2.

3.3.2 Information entropy evaluation: Information entropy is a measurement for the chaotic degree or dispersion degree of distribution (Rusinko and Ivasyuk, 1981). Eigenvalue distribute more dispersed, the smaller value of the information entropy. The more concentrated the information entropy distribution, the larger the information entropy. We use it to evaluate the dispersion of three eigenvalues.

There are three eigenvalues for per point. We should normalize them at first for comparing entropy value convenience. The MinMax scaling normalize equation has been used and defines as:

$$
\eta_{j}^{i}=\frac{\lambda_{j}^{i}-\lambda_{\min }^{i}}{\lambda_{\max }^{i}-\lambda_{\min }^{i}}
$$

where

$$
\begin{aligned}
& i=\text { index of point } \\
& j=\text { index of eigenvalue } \\
& \lambda_{\min }^{i}=\text { minimum value of three eigenvalues } \\
& \lambda_{\max }^{i}=\text { maximum value of three eigenvalues }
\end{aligned}
$$

The information entropy equation is defined as:

$$
\operatorname{Entropy}(i)=\sum_{j=1}^{3}-\eta_{j}^{i} \log _{2}\left(\eta_{j}^{i}\right)
$$

For each point $p_{i}$ in $p[]$, we use $p_{i}$ as centre and radius $a$ make a sphere $s p h_{i}$. Then, compute three eigenvalues of $p_{i}$ from the points in $s p h_{i}$. Apply Eq.(1) and Eq.(2) to get entropy value. At last, label this points joint kind according with Table 2 .

\begin{tabular}{|l|c|}
\hline Entropy value & Labels \\
\hline Entropy $(i) \geq 1.4$ & $L[1]$ \\
$0.9 \leq$ Entropy $(i)<1.4$ & $L[2]$ \\
Entropy $(i)<0.9$ & $L[3]$ \\
\hline
\end{tabular}

Table 2. Entropy value classification

The value 1.4 and 0.9 in table 2 come from the experience in the actual application for extracting power lines.

\section{3D POWER LINE FITTING}

The purpose of 3D power line fitting is to reconstruct the 3D model of power line. The least squares multiply fitting algorithm is introduced for model the power line. Because of power line model is almost in keeping with the catenary curve (Guan et al., 2014). Catenary curve is described by the following equation.

$$
z=a\left(x^{2}+y^{2}\right)+b \sqrt{x^{2}+y^{2}}+c
$$

The polynomial parameters a, b, c should be computed by point cloud data.

\section{RESULTS}

The above depicted method was tested using LongHai Railway point clouds acquired by RIEGL VMX-450 system in Xian, China. The total length of the railway is $11.69 \mathrm{~km}$. The experimental 
platform is microsoft visual studio 2010.

In section 3 , the segment length is $20 \mathrm{~cm}$ in the process of selecting a section to generate seed progress. The volume of bounding box is $10 \mathrm{~cm} 10 \mathrm{~cm} 50 \mathrm{~cm}$. The sphere radius mentioned in section 3.3 is set to be the length of cross sections polygon of bounding box.

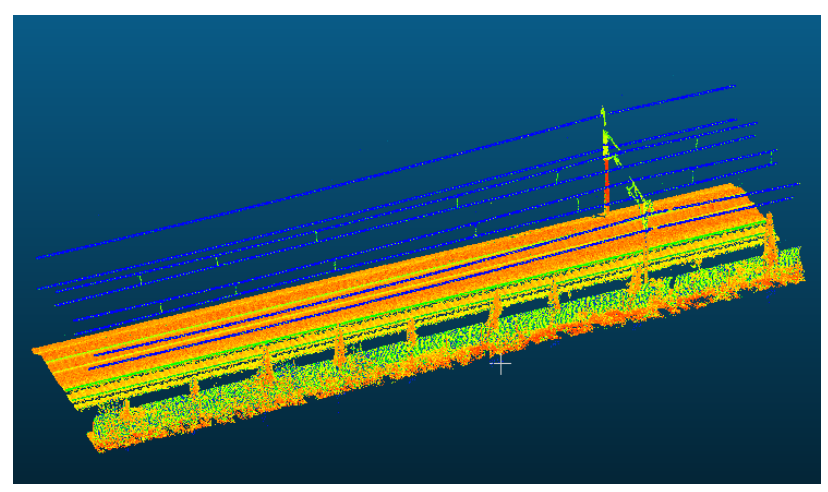

Figure 7. Result of lines detection

As shown in Figure 7, power lines and horizontal suspension lines are separated from vertical suspension lines and electrical poles. The extracted lines are showing in blue. The experimental results demonstrated that power lines can be effectively and robustly extracted from point clouds by our proposed method.

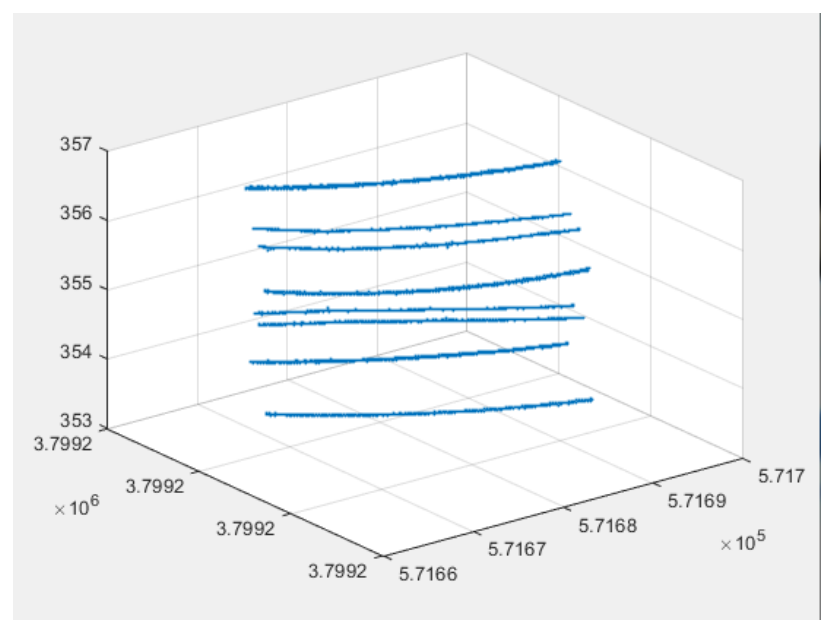

Figure 8. Result of lines detection

Figure 8 shows power lines model in the 3D space. The fitted power line can be well overlapped with the point cloud.

\section{CONCLUSION}

This paper presented an automated, effective power line detection method for extracting power lines in railway surroundings. We utilized the self-adaptive space region growing method to extract power lines. After that use PCA combine with information entropy theory method to judge this region of the power line whether junction or not and which joint kind it belongs to. During the extraction result their fitted models, we conclude that the proposed method performs very well and achieves reliable results. How to use the relationship among power lines and the distance between power line and rail to find out which place violate the railway standard maybe the future work we should do.

\section{ACKNOWLEDGEMENTS}

This work described here is supported by the Xizang Minzu University scientific research project (No. 13myZP03) and Xizang Autonomous Region Natural science fund projects (No. 2015ZR14-16).

\section{REFERENCES}

Chen, D., Zhang, L., Li, J. and Liu, R., 2012. Urban building roof segmentation from airborne lidar point clouds. International journal of remote sensing 33(20), pp. 6497-6515.

Cheng, L., Tong, L., Wang, Y. and Li, M., 2014. Extraction of urban power lines from vehicle-borne lidar data. Remote Sensing 6(4), pp. 3302-3320.

Guan, H., Li, J., Zhou, Y., Yu, Y., Wang, C. and Wen, C., 2014. Automatic extraction of power lines from mobile laser scanning data. In: Geoscience and Remote Sensing Symposium (IGARSS), 2014 IEEE International, IEEE, pp. 918-921.

Guebel, D. V. and Torres, N. V., 2013. Principal component analysis (pca). Encyclopedia of Systems Biology pp. 1739-1743.

Kim, H. B. and Sohn, G., 2013. Point-based classification of power line corridor scene using random forests. Photogrammetric Engineering \& Remote Sensing 79(9), pp. 821-833.

Lehtomäki, M., Jaakkola, A., Hyyppä, J., Kukko, A. and Kaartinen, H., 2010. Detection of vertical pole-like objects in a road environment using vehicle-based laser scanning data. Remote Sensing 2(3), pp. 641-664.

Liu, Y., Li, Z., Hayward, R., Walker, R. and Jin, H., 2009. Classification of airborne lidar intensity data using statistical analysis and hough transform with application to power line corridors. In: Digital Image Computing: Techniques and Applications, 2009. DICTA'09., IEEE, pp. 462-467.

Rusinko, K. and Ivasyuk, V., 1981. Elements of the mathematical theory of inelastic deformation. Materials Science 16(5), pp. $400-405$.

Sohn, G., Jwa, Y. and Kim, H. B., 2012. Automatic powerline scene classification and reconstruction using airborne lidar data. ISPRS Ann. Photogramm. Remote Sens. Spat. Inf. Sci 13(167172), pp. 28. 